\title{
DYNAMIC RE-CLUSTERING LEACH-BASED (DR-LEACH) PROTOCOL FOR WIRELESS SENSOR NETWORKS
}

\author{
Abdallah Ijjeh ${ }^{1}$, Abdalraheem Ijjeh ${ }^{2}$, Huthaifa Al-Issa $^{1}$, Saed Thuneibat ${ }^{1}$ \\ ${ }^{1}$ Department of Electrical and Electronic Engineering, Al-Balqa`Applied University / Al- \\ Huson University College, Irbid, Jordan \\ ${ }^{2}$ Technical college at Ranya, Ranya, Saudi Arabia
}

\begin{abstract}
A Wireless Sensor Network (WSN) contains a large number of sensor nodes equipped with limited energy supplies. In most applications, sensor nodes are deployed in a random fashion. Therefore, battery replacement or charging is considered not practical. As a result, routing protocols must be energy-efficient to prolong the network's lifetime. In this paper, we propose a new Dynamic Re-clustering LEACH-Based protocol (DR-LEACH) which aims to reduce the energy consumption and extending the network's lifetime. The idea is to balance energy consumption of Cluster Heads (CHs) by generating clusters with almost equal number of nodes during each round of the network life time. To perform this, we first calculate the optimal number of $\mathrm{CHs}$ in each round, and based on that we calculate the optimal size of each cluster. Results show that the proposed protocol improves network lifetime and reduces overall energy consumption compared to LEACH and BCDCP protocols.
\end{abstract}

\section{KEYWORDS}

Wireless sensors network (WSN), dynamic clustering, optimal cluster size, energy balancing, network lifetime, residual energy

\section{INTRODUCTION}

Wireless Sensors Networks (WSNs) are widely considered as one of the interesting and rapidly developing fields. They have attracted great attention because of the diverse applications they support in both civilian and military sectors [1]. Typically, a WSN consists of a large number of low-cost, low-power, and multifunctional wireless sensor nodes with sensing, wireless communication and computation capabilities. In many applications, the sensor nodes are randomly deployed. Accordingly, the sensor nodes must organize themselves into a wireless network and cooperate to perform the required task. In addition, WSNs are usually battery powered which means it is very difficult to replace or recharge the batteries as soon as the nodes are deployed [2] [3]. Based on that, many techniques were proposed to achieve longer lifetime and efficient energy consumption. Clustering is one of the effective techniques used to save energy in WSNs [4].Clustering means organizing sensor nodes into different groups called clusters. In each cluster, sensor nodes can be either a Cluster Head $(\mathrm{CH})$ or an ordinary member node. A CH is the group leader in each cluster. It collects sensed data from member nodes, aggregates, and transmits the aggregated data to the next $\mathrm{CH}$ or to the Base Station [5]. The role of an ordinary member node is to sense data from the environment in which they are deployed and send it to the corresponding $\mathrm{CH}$. 


\section{RELATED WORK}

LEACH is one of the most popular clustering techniques used in WSNs to increase the network lifetime [6] [7]. LEACH is an adaptive, self-organizing and distributed clustering protocol. It assumes that the BS is fixed and located far from the sensors, all sensor nodes are homogenous and have limited energy source, sensors can sense the environment at a fixed rate and can communicate among each other, and sensors can directly communicate with BS. The idea of LEACH is to organize the nodes into clusters to distribute the energy among the sensor nodes in the network, and in each cluster there is an elected node called a CH. LEACH introduces the concept of Rounds each of which consists of two phases. Clusters are formed during the set-up phase and data transfer occurs during the steady-state phase.

A centralized routing protocol (contrast to LEACH which is distributed) called Base-Station Controlled Dynamic Clustering Protocol (BCDCP) was introduced in [8]. BCDCP assumes that the BS has sufficient energy during its operation. Furthermore, it assumes that the BS knows the places of all nodes. The fundamental idea of BCDCP is the formation of balanced clusters centrally by the $\mathrm{BS}$, where each $\mathrm{CH}$ manages an equal number of member nodes to avoid $\mathrm{CH}$ overload, and organizing placement of the formed CHs throughout the network area. In BCDCP, there are two phases. The first phase is the setup phase, in which, the BS prepares a list of all potential $\mathrm{CHs}$ based on their remaining energy level. Then, the BS uses the list to pick out the two farthest $\mathrm{CHs}$, and divides the nodes into two groups based on closeness. Then, it performs a balancing process on the groups. This process is reiterated within each group until all clusters are formed. As soon as all clusters are formed, the BS structures a "minimum spanning tree" that links all $\mathrm{CHs}$, and randomly picks out one $\mathrm{CH}$ to forward packets of data to BS. This is in contrast to LEACH where each $\mathrm{CH}$ directly communicates with the BS. The second phase of the $\mathrm{BCDCP}$ protocol is the steady state phase, in which each $\mathrm{CH}$ within each cluster creates a TDMA schedule to minimize collisions between sensor nodes trying to send data to the $\mathrm{CH}$, and the nodes send the sensed data to the $\mathrm{CH}$ which performs data aggregation and compression and route it to the $\mathrm{CH}$ that is responsible for forwarding the data to the $\mathrm{BS}$.

\section{INTRODUCTION TO THE PROPOSED PROTOCOL}

In this paper we propose a Dynamic Re-clustering protocol (DR-LEACH) as an improvement over the LEACH protocol. The main goal is to develop a protocol that optimizes the cluster's size such that each $\mathrm{CH}$ serves an approximately equal number of sensor nodes. Consequently, processing and transmission performed by each $\mathrm{CH}$ is as equal as possible to each other. As a result, network life time will be extended and nodes will die around the same time. The rest of the paper is organized as follows: Section 2 explains the network and radio models assumed in our protocol. A detailed description of the Methodology of DC-LEACH is explained in section 3. Section 4 demonstrates the experimental of the DC-LEACH. Finally we conclude the paper in section 5 .

\section{NETWORK ASSUMPTIONS AND RADIO MODEL}

In this section we describe the network and radio model assumed in the DR-LEACH. We did use the first radio model used in the original LEACH protocol [6]. The model assumes the following:

- The BS is fixed during the running of the protocol (immobile).

- All sensor nodes are homogenous and supplied with the same initial energy.

- All sensor nodes are stationary. 
In addition, we assume that each sensor node is capable to act in two different roles: $\mathrm{CH}$ role and normal sensing role. When the node is in the normal sensing role, it senses the environment and sends the sensed data to its $\mathrm{CH}$. In the $\mathrm{CH}$ role, it collects the sensed data from its members in the cluster, performs data aggregation and compression, then generates a composite signal and transmits to the BS.

Figure 1 shows the first order radio model. The required energy to transmit and receive a k-bit data message over a distanced $d$ is given by (2) and (3):

$$
\begin{gathered}
E_{T}(k, r)=\left\{\begin{array}{c}
E_{T X} * k+\varepsilon_{f s} * d^{2} * k i f d \leq d_{0} \\
E_{T X} * k+\varepsilon_{m p} * d^{4} * k i f d>d_{0}
\end{array}\right. \\
E_{R}=E_{R X} * k
\end{gathered}
$$

Where $\mathrm{E}_{\mathrm{TX}}$ and $\mathrm{E}_{\mathrm{RX}}$ are the required energy consumed per bit to operate the transmitter or receiver circuitry, respectively. $E_{\mathrm{R}}$ is the required energy to receive k-bit data message. $\varepsilon_{\mathrm{fs}}$ and $\varepsilon_{\mathrm{mp}}$ are amplifier parameters correspond to free space and multi-path fading models. $d_{0}$ is the threshold distance given by :

$$
d_{0}=\sqrt{\frac{\varepsilon_{\mathrm{fs}}}{\varepsilon_{\mathrm{mp}}}}
$$

For the purpose of simulations we considered that $\mathrm{E}_{\mathrm{TX}}=\mathrm{E}_{\mathrm{RX}}=50 \mathrm{~nJ} / \mathrm{bit}, \varepsilon_{\mathrm{fs}}=10 \mathrm{pJ} / \mathrm{b} / \mathrm{m}^{2}$, $\varepsilon_{\mathrm{mp}}=0.0013 \mathrm{pJ} / \mathrm{b} / \mathrm{m}^{4} . \mathrm{E}_{\mathrm{DA}}=5 \mathrm{~nJ} / \mathrm{b} /$ message, where $\mathrm{E}_{\mathrm{DA}}$ is the energy required for data aggregation. These values are mostly used in the literature for evaluating LEACH performance $[8,9,10,11]$.

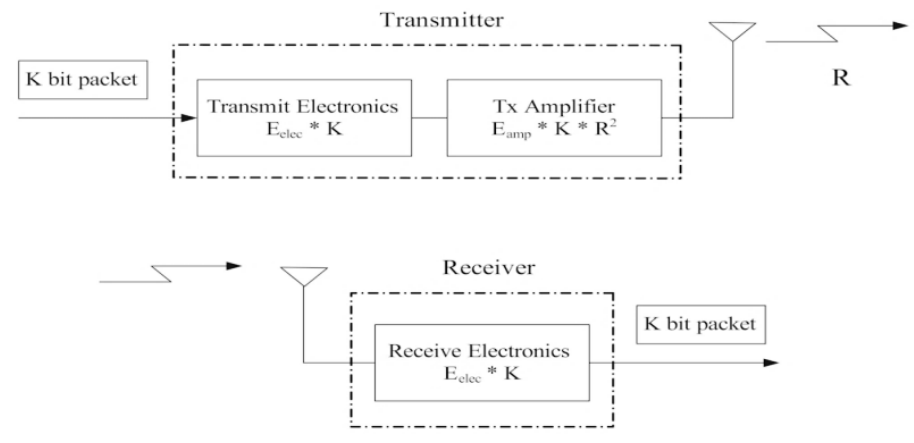

Figure 1. First order radio model. [12]

\section{METHODOLOGY OF THE PROPOSED PROTOCOL}

The original LEACH is composed of two phases: the setup phase and the steady-state phase. The proposed protocol, DR-LEACH, is also composed of same two phases. However, it added two more steps in the setup phase: splitting and merging. This section provides a detailed description of the proposed protocol phases.

\subsection{DR-LEACH SETUP PHASE}

At the beginning of the setup phase, initial $\mathrm{CHs}$ are elected and clusters are formed similar to the LEACH protocol. The formed clusters are not optimal in sizes, i.e. some clusters might have very small number of nodes while some might have large number of nodes. As a result, $\mathrm{CHs}$ which serves a large number of sensor nodes will consume more energy and will die faster than others. 
This affects the functionality of the network in the sense that some clusters might get isolated from the network due to fast discharge of the $\mathrm{CH}$. In addition, it also affects the efficiency of energy consumption and the network lifetime. DR-LEACH tries to distribute nodes among CHs equally such that the CH's have equal amount of work to do in terms of processing and transmission. This will improve the efficiency of the energy consumption and as a result prolonging the network lifetime.

After the initial clusters are formed, we calculate the optimal number of clusters $\left(\mathrm{K}_{\mathrm{opt}}\right)[13] . \mathrm{K}_{\mathrm{opt}}$ is calculated as shown in (5)[14]:

$$
K_{\text {opt }}=\frac{(0.5 \times n \times \text { thresholddistance } \times \pi) \times \text { one dimensionof thefield }}{(D b s)^{2}}
$$

Where $\mathrm{n}$ is the number of alive nodes and $\mathrm{D}_{\mathrm{bs}}$ is the average distance between the $\mathrm{CH}$ and BS. $\mathrm{D}_{\mathrm{bs}}$ is calculated as shown in (6)[14]:

$$
\mathrm{D}_{\mathrm{bs}}=(0.765 * \text { one dimension of the field }) / 2
$$

Then, we calculate the optimal cluster size $\left(\mathrm{N}_{\mathrm{opt}}\right)$ by dividing the total number of alive nodes, $\mathrm{n}$, over the previously computed $\mathrm{K}_{\mathrm{opt}}$. That is, $\mathrm{N}_{\mathrm{opt}}$ is calculated by:

$$
N_{\text {opt }}=\frac{n(\text { alive nodes) }}{K_{\text {opt }}}
$$

For the best result, the size of each cluster should be exactly $\mathrm{N}_{\text {opt }}$. However, this requires more computation to guarantee this exact size since a size which is one more or one less than the optimal size will not be considered optimal and hence we have to reprocess the cluster until we get an exact match which will force the protocol to converge after longer time (provided that the total number of alive nose is an exact multiple of $\mathrm{K}_{\mathrm{opt}}$. Otherwise, the protocol will not converge). Therefore, to let the protocol converge faster and reduce processing, DR-LEACH considers a cluster to be optimal if its size lies in an interval that is bound by an upper and lower limits and is centered at $\mathrm{N}_{\mathrm{opt}}$ as shown in Figure 2. This interval is called the optimal range of the cluster size. Consequently, if the cluster size is greater than the minimum limit $\left(\mathrm{N}_{\text {opt_min }}\right)$ and less than the maximum limit $\left(\mathrm{N}_{\text {opt_max }}\right.$ ), it is considered as an optimal cluster size. Otherwise, we have to perform re-clustering: splitting large clusters or merging small cluster to form an optimal cluster.

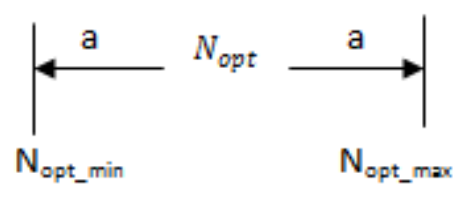

Figure 2. Optimal cluster size boundaries

$\mathrm{N}_{\text {opt_max, }} \mathrm{N}_{\text {opt_min }}$ are calculated as shown in (8) and (9), respectively

$$
\begin{aligned}
& N_{\text {opt_max }}=N_{o p t}+a \\
& N_{\text {opt_min }}=N_{\text {opt }}-a
\end{aligned}
$$


Where $a$ is a dummy variable that represent the deviation from the exact optimal cluster size.

For faster convergence and less computation, we assume that $\mathrm{N}_{\text {opt_max }}$ is twice larger than $\mathrm{N}_{\text {opt_min }}$ such that if we split a large cluster in half, it would result in a two clusters whose sizes are optimal and need no further processing is required. This is shown in (10).

$$
N_{\text {opt_max }}=2 * N_{\text {opt_min }}
$$

Using equations (8), (9), and (10), we can compute the values of $\mathrm{N}_{\text {opt_min }}$ and $\mathrm{N}_{\text {opt_max }}$ as shown in (11) and (12), respectively.

$$
\begin{aligned}
& N_{o p t \_m i n}=\frac{2}{3} \times N_{o p t} \\
& N_{o p t \_m a x}=\frac{4}{3} \times N_{o p t}
\end{aligned}
$$

As soon as $\mathrm{N}_{\text {opt_max }}$ and $\mathrm{N}_{\text {opt_min }}$ are calculated, the nest step in the setup phase is that each cluster checks its size. If the cluster size is less than $\mathrm{N}_{\text {opt_min }}$ we perform merging. In this step, the $\mathrm{CH}$ informs its members that it is no longer a $\mathrm{CH}$, and returns to the normal sensing node. Each detached member node independently joins the closest $\mathrm{CH}$ (a one with the highest RSSI signal). If, as a result of merging, a cluster's size becomes greater than $\mathrm{N}_{\text {opt_max }}$, it is split as described in the next paragraph. Otherwise, it is still in the optimal and no further processing is required. Each time a merging operation is performed, the number of clusters is decreased by 1 .

A splitting operation is performed if the cluster size exceeds $\mathrm{N}_{\text {opt_max }}$. In such case, the $\mathrm{CH}$ searches its cluster for a node with the highest residual energy. When the $\mathrm{CH}$ finds the desired node, it sends a signal message to that node to inform it to become a second $\mathrm{CH}\left(\mathrm{CH}_{\mathrm{nd}}\right)$ within the cluster. Then, each node of the cluster joins either of the CH's based on the RSSI. As a result, the old cluster (whose size is greater than Nopt_max) will be split into two clusters. In the case that one of the resulting clusters has a size that is less than $\mathrm{N}_{\text {opt_min }}$, merging is performed as described in the above paragraph. Each time a splitting operation is done the number of clusters is increased by 1 .

The merging and splitting operations are repeatedly performed for all clusters to optimize their sizes. At the end, all formed clusters are between $\mathrm{N}_{\text {opt_min }}$ and $\mathrm{N}_{\text {opt_max }}$, which means are optimal range and no further processing in regard of forming clusters is required.

\subsection{DC-LEACH STEADY STATE PHASE}

As soon as all clusters are formed, each $\mathrm{CH}$ creates a TDMA schedule to organize the transmissions of its member nodes. A member node starts sending its sensed data to the $\mathrm{CH}$ during its allocated time slot. When the $\mathrm{CH}$ receives all data from its members, $\mathrm{CH}$ performs data aggregation and data compression then it generates a composite signal and transmitted to the BS. In fact, this is similar to the original LEACH steady state phase. In Figure 3 we illustrate the methodology of our proposed protocol. 


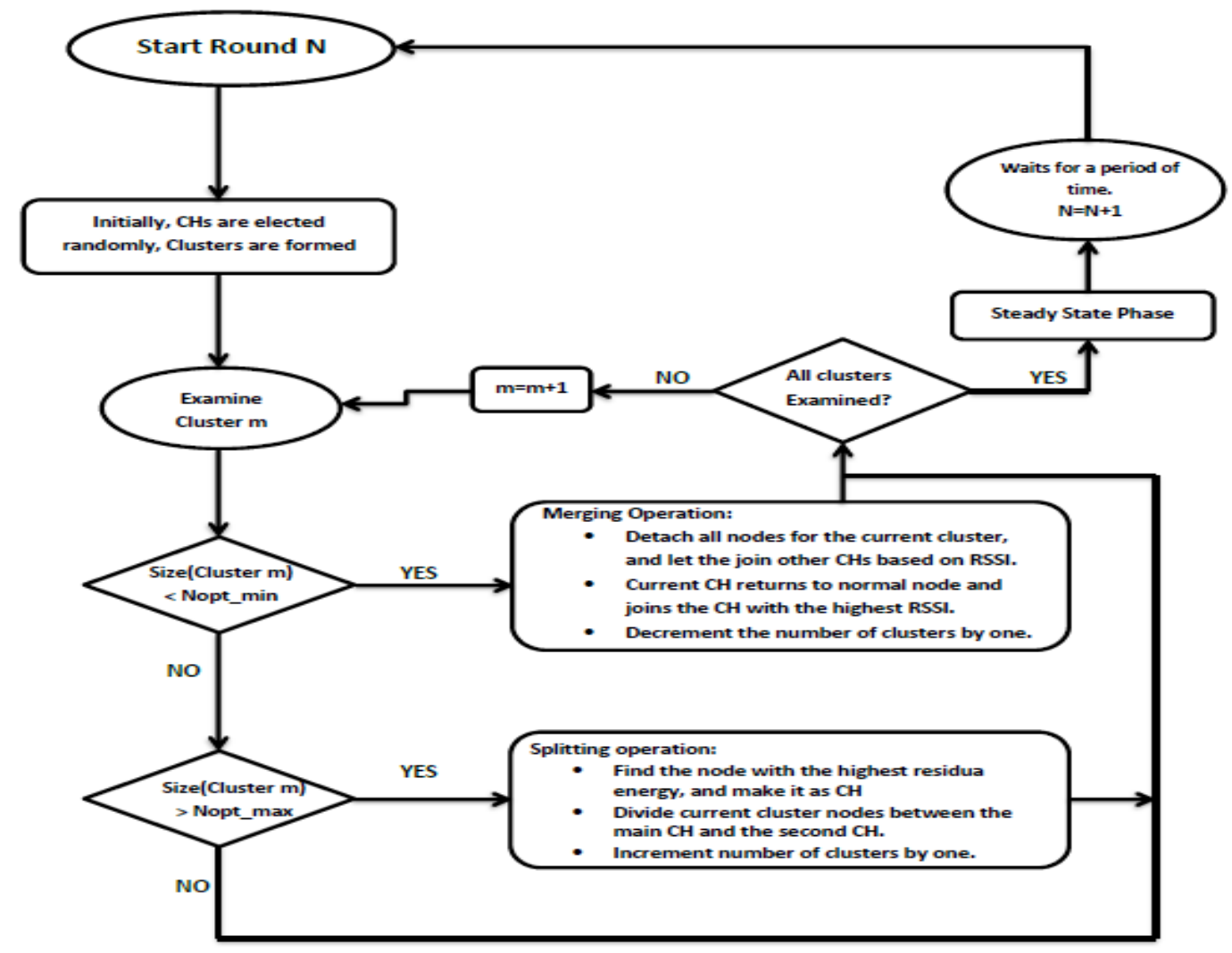

Figure 3: Illustration of the proposed protocol methodology.

\section{EXPERIMENTAL RESULTS}

In this section, the experiment results and performance of the DR-LEACH protocol are presented. The protocol is simulated using MATLAB as were done in [8,6]. Each run of the protocol is repeated 20 times and the results are averaged to guarantee statistical reliability. After that, the results are compared to both BCDCP and LEACH protocols. The performance is measured against the number of alive nodes, total residual energy, first dead node, and $50 \%$ dead nodes. Table 1 lists the values of the parameters used in the simulation, which are typically used for evaluating LEACH performance $[8,9,10,11]$.

Table 1. List of parameters

\begin{tabular}{|c|c|}
\hline The Parameter & The Value \\
\hline Data Packet Size & $2000 \mathrm{bits}$ \\
\hline Initial Energy & $0.5 \mathrm{~J}$ \\
\hline$E_{\text {elec }}$ & $50 \mathrm{~nJ} / \mathrm{bit}$ \\
\hline$E_{m p}$ & $0.0013 \mathrm{pJ} / \mathrm{b} / \mathrm{m}^{4}$ \\
\hline$E_{f s}$ & $10 \mathrm{pJ} / \mathrm{bit} / \mathrm{m}^{2}$ \\
\hline$E_{D A}$ & $5 \mathrm{~nJ} / \mathrm{b} / \mathrm{message}$ \\
\hline Deployment & Random \\
\hline
\end{tabular}


In the experiment we simulate a 300 sensor nodes deployed in an area of $(100 \times 100) \mathrm{m}$. Different locations of the BS are considered in this experiment. Specifically, the BS is positions at $(0,0)$ [lower left corner], $(0,-100),(0,-200)$, and $(150,50)$.

Figures 4, 5, 6, and 7 show the number of alive sensor nodes in each round of the protocols for different positions of the BS. As can be depicted from the figure, DR-LEACH is the most energysaving protocol for the first dead node, and $50 \%$ dead nodes and the last dead node whether the BS is close or far from the nodes. As a result, DR-LEACH extends the network lifetime compared to LEACH and BCDCP protocols regardless of the BS location. Hence, it shows more scalability in terms of how far the BS can be away from the nodes. All of this is due to the way it handle cluster formation and generating optimal clusters.

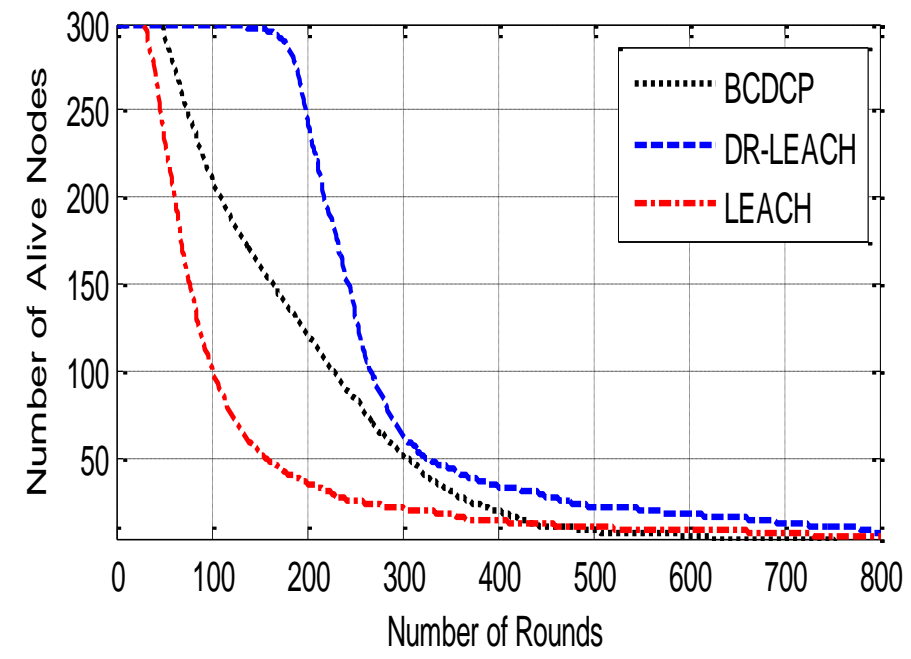

Figure 4. Number of live nodes for the DR-LEACH, BCDCP and LEACH, BS at $(0,0)$

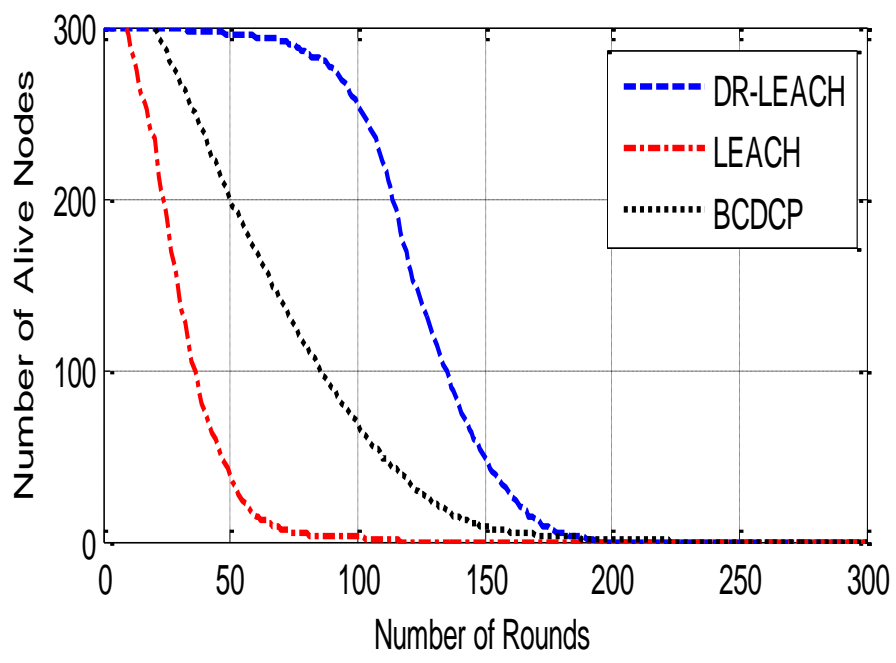

Figure 5. Number of live nodes for the DR-LEACH, BCDCP and LEACH, BS at $(0,-100)$ 


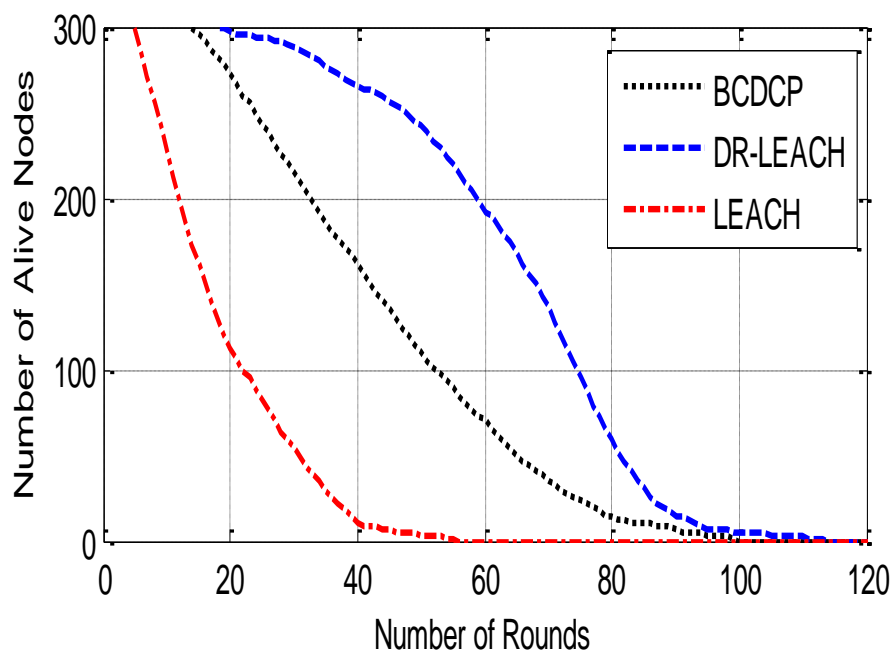

Figure 6. Number of live nodes for the DR-LEACH, BCDCP and LEACH, BS at (0-200)

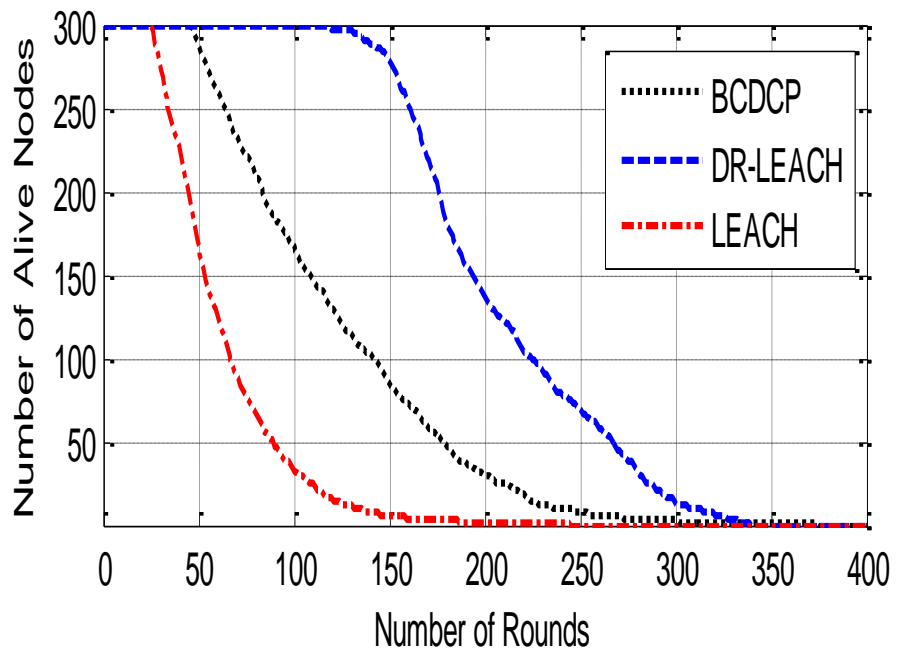

Figure 7. Number of live nodes for the DR-LEACH, BCDCP and LEACH, BS at $(150,50)$

Figures $8,9,10$, and 11 show the total residual energy during each round of DR-LEACH, LEACH, and BCDCP protocols where the BS is located at $(0,0),(0,-100),(0,-200)$, and $(150$, 50) respectively. It can be seen that DR-LEACH is more energy-conserving than the other two protocols. This is due primarily to the dynamic merging and splitting mechanisms it utilizes. This leads to balanced energy consumption between clusters. Consequently, less energy is consumed in each round by a cluster. As a result, more energy is conserved in each round, and the network lifetime is extended, whether for the first dead node, $50 \%$ dead node, or the last dead node 
International Journal of Computer Networks \& Communications (IJCNC) Vol.7, No.6, November 2015

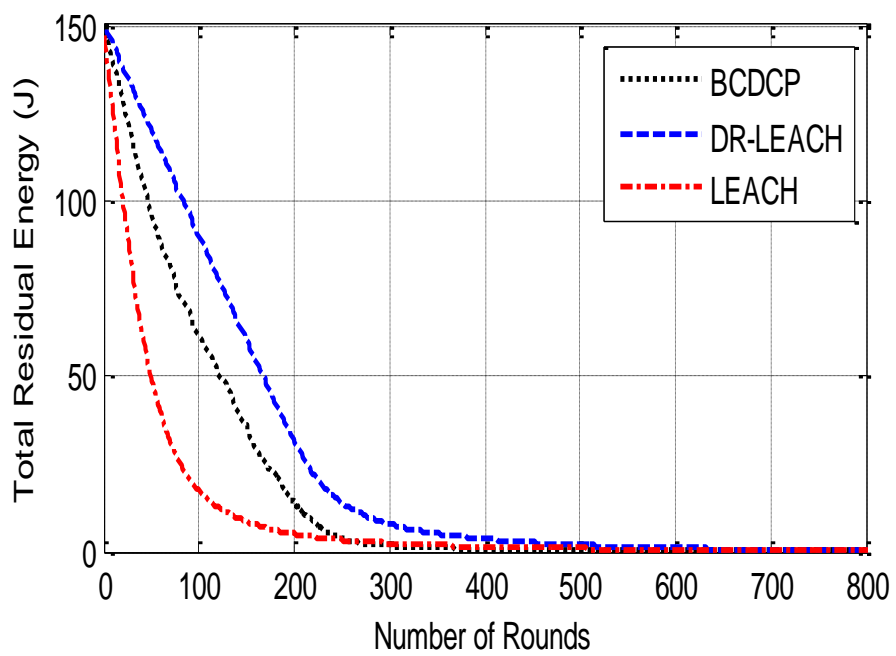

Figure 8. Total residual energy for the alive nodes for DR-LEACH, BCDCP and LEACH, BS at $(0,0)$

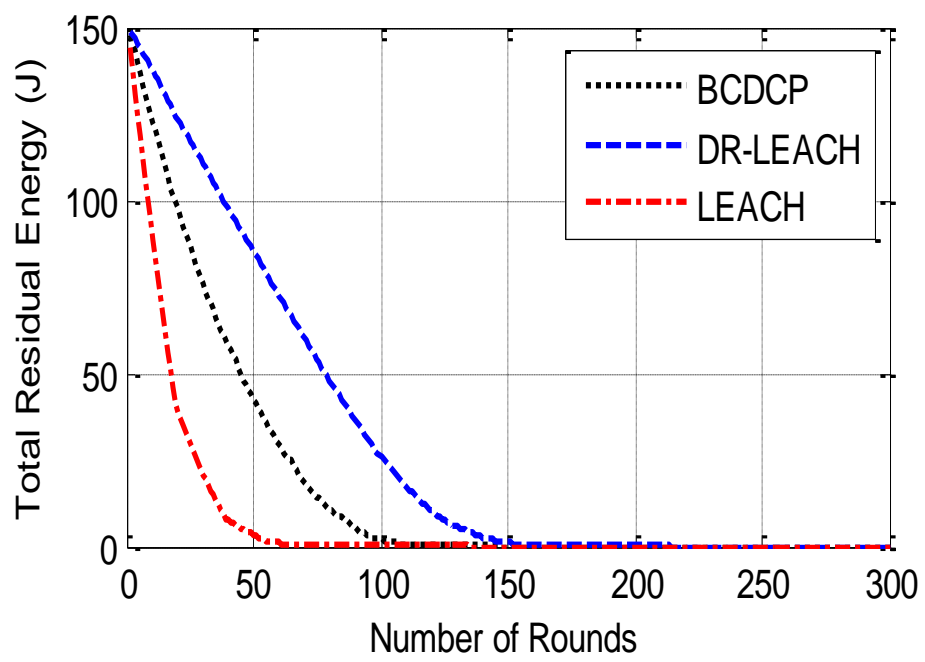

Figure 9: Total residual energy for the alive nodes for DR-LEACH, BCDCP and LEACH, BS at $(0,-100)$

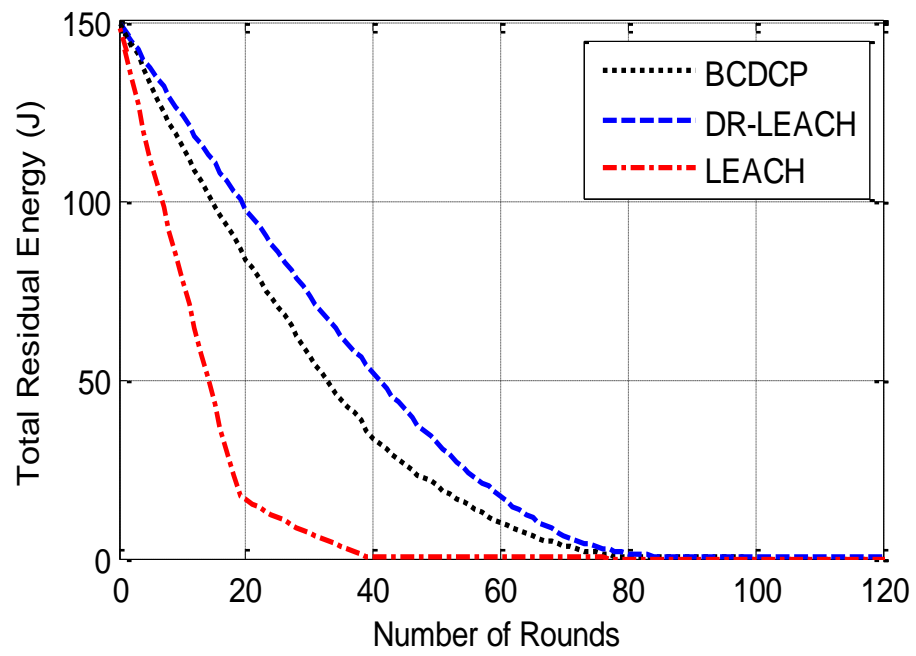

Figure 10: Total residual energy for the alive nodes for DR-LEACH, BCDCP and LEACH, BS at $(0,-200)$ 


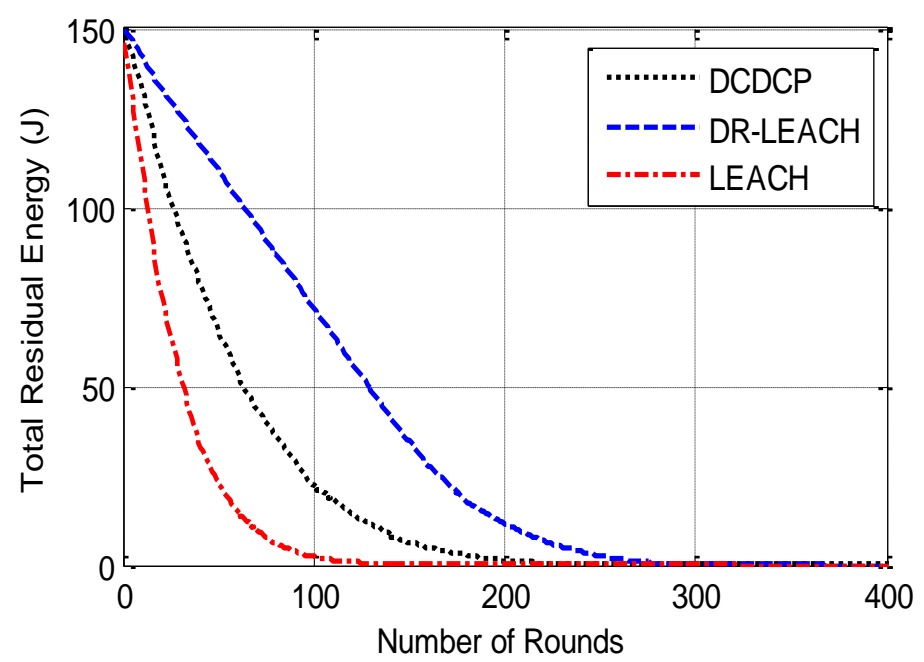

Figure 11: Total residual energy for the alive nodes for DR-LEACH, BCDCP and LEACH, BS at $(150,50)$

Table 2 summarizes the results of the experiment and provides a comparison of network lifetimes for the first dead node, $50 \%$ dead node, and the enhancement percentage of DR-LEACH over $\mathrm{BCDCP}$ and LEACH protocols. As can be figured out from the table and the above figures, the location of the BS greatly affects the occurrence rate of the first dead node. Farther BS will result in consuming more energy. However, DR-LEACH performance is still better than the other protocols regardless of the location of the BS. For example, when the BS is at $(0,-100)$ the DCLEACH is $200 \%$ and $300 \%$ better than LEACH in terms of first dead node and $50 \%$ dead nodes, respectively. In addition, it is $36.4 \%$ and $79.1 \%$ better than the BCDCP in terms of first dead node and $50 \%$ dead nodes respectively at the same location.

Table2: Comparison of network lifetimes for the DC-LEACH, BCDCP and LEACH protocols for different BS locations (No of sensor nodes $=300$, area $=100 \mathrm{~m} \times 100 \mathrm{~m}$ ).

\begin{tabular}{|c|c|c|c|c|c|c|}
\hline \multirow[t]{2}{*}{$\begin{array}{l}\text { BS } \\
\text { Location }\end{array}$} & \multirow[t]{2}{*}{ Protocol } & \multicolumn{2}{|c|}{$\begin{array}{l}\text { Round at which the event } \\
\text { happened }\end{array}$} & \multicolumn{3}{|c|}{$\begin{array}{l}\text { Enhancement \% of DR- } \\
\text { LEACH compared to other } \\
\text { protocols }\end{array}$} \\
\hline & & $\begin{array}{l}\text { First Dead } \\
\text { Node }\end{array}$ & $\begin{array}{l}50 \% \text { Dead } \\
\text { Nodes }\end{array}$ & $\begin{array}{l}\text { First Dead } \\
\text { Node }\end{array}$ & $\begin{array}{l}50 \% \\
\text { Nodes }\end{array}$ & Dead \\
\hline \multirow[t]{3}{*}{$(0,0) \mathrm{m}$} & DR-LEACH & 107 & 242 & - & - & \\
\hline & BCDCP & 46 & 165 & $132.6 \%$ & $46.67 \%$ & \\
\hline & LEACH & 25 & 77 & $328 \%$ & $214.3 \%$ & \\
\hline \multirow[t]{3}{*}{$(0,-100) \mathrm{m}$} & DR-LEACH & 30 & 120 & - & - & \\
\hline & BCDCP & 22 & 67 & $36.4 \%$ & $79.1 \%$ & \\
\hline & LEACH & 10 & 30 & $200 \%$ & $300 \%$ & \\
\hline \multirow[t]{3}{*}{$(0,-200) \mathrm{m}$} & DR-LEACH & 18 & 68 & - & - & \\
\hline & BCDCP & 14 & 42 & $28.57 \%$ & $61.9 \%$ & \\
\hline & LEACH & 6 & 18 & $200 \%$ & $277.78 \%$ & \\
\hline \multirow[t]{3}{*}{$(150,50) \mathrm{m}$} & DR-LEACH & 50 & 194 & - & - & \\
\hline & BCDCP & 46 & 109 & $8.69 \%$ & $77.98 \%$ & \\
\hline & LEACH & 26 & 54 & $92.3 \%$ & $259.25 \%$ & \\
\hline
\end{tabular}




\section{CONCLUSIONS}

This paper presents a dynamic re-clustering protocol (DR-LEACH) to balance the load among $\mathrm{CHs}$ by taking into consideration the optimal number of $\mathrm{CHs}$ and calculating the optimal cluster size. Based on that, the protocol re-clusters all clusters that beneath lower boundary of optimal cluster size and split clusters that are above the upper optimal boundary. Although these techniques will increase up the computational power, but this rise is always negligible compared to the reduction in number of required transmissions. In this paper we have simulated the proposed protocol and compared it with BCDCP and LEACH protocols. Simulation results show that in DR-LEACH is better than both LEACH and BCDCP. In fact, DR-LEACH shows great improvement over LEACH regardless of the location of the BS. For example, an enhancement as large as $328 \%$ is achieved when the BS is located at $(0,0)$, and as minimum as $92.3 \%$ when the BS is located at $(150,50)$. Also, Improvement over the BCDCP is achieved but in less figures. In addition, simulation results show that DR-LEACH is more energy-conserving than both LEACH and $\mathrm{BCDCP}$ protocols irrespective of the BS location. In addition, it can be concluded that the technique of cluster balancing in DR-LEACH is better than the one used in BCDCP since DRLEACH is more energy conserving. In $\mathrm{BCDCP}$, there is a single $\mathrm{CH}$ which is randomly chosen to forward data the BS. All the $\mathrm{CHs}$ send their data to that chosen node which will finally send the aggregated data to the BS. As a result, energy consumption is not balanced between nodes. On the other hand, creating equal clusters that directly communicate with the BS distributes energy consumption more evenly which results in having more residual energy in the network. As a result, the network lifetime is extended and the nodes keep running for longer time and die relatively close in time to each other.

\section{REFERENCES}

[1] Deborah Estrin et al, "Next century challenges: scalable coordination in sensor networks," in ACM/IEEE international conference on Mobile computing and networking, New York, NY, USA, 1999 .

[2] C. F. Wang, J. D. Shih, B. H. Pan and T. Y. Wu, "A Network Lifetime Enhancement Method for Sink Relocation and Its Analysis in Wireless Sensor Networks," Sensors Journal, IEEE (Volume:14, Issue: 6 ), pp. 1932 - 1943, 2015.

[3] J. Abawajy and S. Ghanavati, "An Alternative Node Deployment Scheme for WSNs," Sensors Journal, IEEE (Volume:15 , Issue: 2 ), pp. 667 - 675, 2015.

[4] Guan Xin et al, "EEHCA: An Energy-Efficient Hierarchical Clustering Algorithm for Wireless Sensor Networks," vol. 8, no. 2, 2008.

[5] S. Ghiasi et al, "Optimal Energy Aware Clustering in Sensor Networks," MDPI Sensors, vol. 2, no. 7, p. 258-269, 2002.

[6] W. R. Heinzelman, A. Chandrakasan and H. Balakrishnan, "Energy-efficient communication protocol for wireless microsensor networks," in Proceedings of the 33rd Annual Hawaii International Conference on System Sciences, 2000. , Hawaii , 2000.

[7] M. Aslam et al, "Survey of Extended LEACH-Based Clustering Routing Protocols for Wireless Sensor Networks," arXiv preprint arXiv:1207.2609, vol. v1, 2012.

[8] S. D. Muruganathan, D. C. F. Ma and R. I. Bh, "A centralized energy-efficient routing protocol for wireless sensor networks," Communications Magazine, IEEE, vol. 43, no. 13, pp. S8-S13, March 2005.

[9] T. Kang, J. Yun, H. Lee and I. Lee, "A Clustering Method for Energy Efficient Routing in Wireless Sensor Networks," in International Conference on Electronics, Hardware, Wireless and Optical Communications EHAC'07 Proceedings of the 6th WSEAS, Corfu Island, Greece, 2007.

[10] M.Bani Yassein et al, "Improvement on LEACH Protocol of Wireless Sensor Network (VLEACH)," in Journal of Digital Content Technology and its Applications, 2009.

[11] M. Tong and M. Tang, "LEACH-B: An Improved LEACH Protocol for Wireless Sensor Network," in 6th International Conference on wireless Communications Networking and Mobile Computing (WiCOM), Chengdu, 2010. 
[12] C. Y. Wen and Y. C. Chen, "Dynamic Hierarchical Sleep Scheduling for Wireless Ad-Hoc Sensor Networks," vol. 9, no. 5, 2009.

[13] A.B.M. Alim Al Islam et al, "Finding the Optimal Percentage of Cluster Heads from a New and Complete Mathematical Model on LEACH," Scientific Research, vol. 2, no. 2, pp. 129-140, 2010.

[14] V. S. Krishna Gopal Vijayvargiya, "An Amend Implementation on LEACH protocol based on Energy Hierarchy," International Journal of Current Engineering and Technology, vol. 2, no. 4, pp. 427-431, 2012.

[15] W.R. Heinzelman et al, "Energy-efficient communication protocol for wireless microsensor networks," in System Sciences, 2000. Proceedings of the 33rd Annual Hawaii International Conference on, 2000..

\section{AUTHORS}

Dr. Abdullah Ijjeh has received his BSc in Electrical Engineering from Kuwait University in 1981. He received his M.Sc. in communication engineering from Yarmouk University, Jordan in 1988. He received his $\mathrm{PhD}$ in communication engineering from The BonchBruevich Saint - Petersburg State University of Telecommunications, St. Petersburg, Russia 1995. Currently he is working at the Department of communication engineering at AlBalqa Applied University, Jordan. His research interests are fibre optics and wireless and

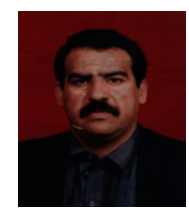
communication systems.

Abdalraheem Ijjeh has obtained his BSc form Yrmouk university 2009, M.Sc from the Jordanian 2013. Currently he is a lecturer in the Technical College at Ranya, Ranya, Saudi Arabia

Dr. Huthaifa Al-Issa is currently working as an assistant professor at the department of communication engineering at Al-Balqa` Applied University, Jordan.

Dr. Saed Thuneibat is currently working as an associate professor at the department of communication engineering at Al-Balqa` Applied University, Jordan. 